\title{
Fototerapia en combinación con otras terapias
}

\section{en psoriasis}

\author{
Phototherapy in combination with other therapies in psoriasis
}

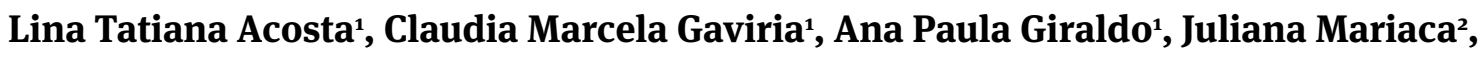 Melisa Montes ${ }^{2}$, Sol Beatriz Jiménez ${ }^{3}$}

1. Médica, residente de Dermatología, Universidad CES, Medellín, Colombia

2. Médica, residente de Dermatología, Universidad Pontificia Bolivariana, Medellín, Colombia

3. Médica dermatóloga; jefe, Servicio de Dermatología, Grupo Dermatología CES, Universidad CES, Medellín, Colombia

\section{RESUMEN}

La psoriasis es una enfermedad crónica inflamatoria, multifactorial, que afecta del 1 al $3 \%$ de la población mundial. Para su tratamiento se ha descrito un sinnúmero de esquemas terapéuticos, entre los cuales se encuentran los tratamientos tópicos combinados para la psoriasis leve a moderada, mientras que la psoriasis grave requiere un manejo más completo con fototerapia y tratamientos sistémicos, como retinoides orales, metotrexato, ciclosporina y medicamentos biológicos.

Aunque muchos datos apoyan la eficacia de la fototerapia en el tratamiento de la psoriasis, aún hay gran variabilidad en su aplicación en todo el mundo y cada vez más se apoya el concepto de tratamientos combinados, los cuales pueden aumentar la eficacia y la tolerabilidad, e inclusive, pueden disminuir los efectos secundarios de ciertos medicamentos aumentando el perfil de seguridad del tratamiento.

Sin embargo, a pesar de lo anterior, la manera como la fototerapia ha encajado en el uso combinado con otros agentes, tópicos o sistémicos, apenas está siendo dilucidada.

PALABRAS CLAVE: psoriasis, fototerapia, retinoides, metotrexato, productos biológicos.

\section{SUMMARY}

Psoriasis is a multifactorial chronic inflammatory disease that affects 1 to $3 \%$ of the world population. For treatment they have been described a number of therapeutic regimens in which are combined for mild to moderate psoriasis topical therapies, while for severe psoriasis requires more aggressive treatment with phototherapy and/or systemic treatments like oral retinoids, methotrexate, cyclosporine and biological medicines. Although many data support the efficacy of phototherapy in the treatment of psoriasis, there is still great variability in their application worldwide and increasingly the concept of combination therapies is supported; which can increase the efficacy, tolerability and can even reduce side effects of certain medications increase the safety profile of treatment. However, despite the above, how phototherapy conceded in the combined use with other agents, topical or systemic, just being elucidated.

KEY WORDS: Psoriasis, phototherapy, retinoids, methotrexate, Biologics.

\author{
Correspondencia: \\ Claudia Marcela Gaviria \\ Email: \\ claugavio2@hotmail.com \\ Recibido: 12 de mayo de 2015 \\ Aceptado: 13 de abril de 2016 \\ Conflictos de intereses. \\ Sol Beatriz Jiménez ha sido \\ conferencista para los labora- \\ torios Pfizer, Novartis, AbbVie y \\ Janssen, y labora en la Unidad \\ de Dermatología de la Univer- \\ sidad CES, donde funciona la \\ Unidad de Fototerapia, pero no \\ recibe honorarios adicionales $\mathrm{ni}$ \\ dividendos por esta actividad.
}




\section{INTRODUCCIÓN}

La psoriasis es una enfermedad crónica inflamatoria, multifactorial, que afecta el 1 al $3 \%$ de la población mundial $^{1,2}$. Se caracteriza clásicamente por áreas engrosadas de piel eritematosa brillante, cubierta con escamas de color blanco a plateado, que compromete la piel y, en algunos casos, las mucosas, las uñas y las articulaciones; todo esto es producido por anormalidades en la proliferación y la diferenciación de los queratinocitos ${ }^{3,4}$.

Desde la descripción de la enfermedad hasta el día de hoy, se han utilizado múltiples métodos de clasificación para la psoriasis, de acuerdo con sus características clínicas, el porcentaje de superficie corporal comprometida y la seriedad de las lesiones. Las escalas más usadas son: el índice de gravedad del área de psoriasis (Psoriasis Area Severity Index, PASI), el porcentaje del área de superficie corporal comprometida (Body Surface Area, BSA) y el índice de calidad de vida en dermatología (Dermatologic Life Quality Index, DLQI)².

La psoriasis también se puede considerar grave cuando compromete las palmas, las plantas o ambas, ya que puede llevar a discapacidad funcional. Se estima que, aproximadamente, el $30 \%$ de las psoriasis son moderadas a graves, y esto acarrea un impacto profundamente negativo en la calidad de vida del paciente ${ }^{4}$.

En la práctica clínica se usan frecuentemente tratamientos tópicos combinados para la psoriasis leve a moderada, mientras que la grave requiere fototerapia, tratamientos sistémicos o ambos, incluidos entre estos últimos los retinoides orales de tipo acitretín, el metotrexato, la ciclosporina y los productos biológicos ${ }^{1,3}$.

La fototerapia, en forma de luz solar natural, se ha usado durante miles de años para el tratamiento de numerosas enfermedades de la piel. Aunque muchos datos apoyan la eficacia de la fototerapia en el tratamiento de la psoriasis, aún hay gran variabilidad en su aplicación en todo el mundo. La manera como la fototerapia con radiación ultravioleta $B$ (UVB) ha encajado en el uso combinado con otros agentes, tópicos o sistémicos, apenas está siendo dilucidada ${ }^{1}$.

\section{Fototerapia más tratamiento tópico}

Aproximadamente, el $80 \%$ de los pacientes con psoriasis tiene enfermedad limitada o leve (PASI, BSA y DLQI menores o iguales a $10 \%$ y pueden ser tratados con agentes tópicos de forma eficaz y segura. En las psoriasis más extensas que requieren fototerapia o productos biológicos, los medicamentos tópicos pueden usarse conjuntamente. Sin embargo, no se recomiendan de forma rutinaria los agentes tópicos como monoterapia en caso de una psoriasis con compromiso extenso o persistente, teniendo en cuenta que se requerirían altas dosis de medicamento, lo cual ocasionaría un mayor riesgo de efectos secundarios y de abandono del tratamiento.

El tratamiento debe ser individualizado según las necesidades, expectativas y características particulares en cada caso, como gravedad, localización y edad de paciente, entre otras ${ }^{5,6}$.

\section{PUVA (psoraleno y radiación UVA) más esteroides tópicos}

A pesar de que los esteroides tópicos son la piedra angular en el tratamiento de la mayoría de las psoriasis, especialmente aquellas con compromiso limitado, la monoterapia no se recomienda cuando el compromiso es grave.

En este caso, los esteroides tópicos se usan como coadyuvantes en otro tipo de terapias, entre las cuales se encuentra la fototerapia. Entre sus mecanismos de acción se incluyen efectos antiinflamatorios, antiproliferativos, inmunosupresores y vasoconstrictores ${ }^{5}$ .Los esteroides tópicos pueden combinarse de manera segura con fotoquimioterapia para el tratamiento de brotes agudos, pero requieren su disminución progresiva una vez alcanzada la remisión de las lesiones. para evitar taquifilaxia ${ }^{2,6}$.

Los estudios han demostrado que la combinación favorece la remisión en más corto tiempo y disminuye la dosis de radiación UVA acumulada requerida. Sin embargo, en algunos estudios se evidencia acortamiento en la duración de la fase de remisión ${ }^{7-9}$.

\section{Radiación UVB más esteroides tópicos}

El uso concomitante de esteroides tópicos con radiación ultravioleta B (UVB) disminuye inicialmente el grosor de la placa de psoriasis, alcanzándose una resolución más rápida de las lesiones; sin embargo, su uso combinado no reduce la dosis de UVB y, por el contrario, se ha encontrado una asociación con mayores tasas de recaídas y no se ha demostrado beneficio a largo plazo, por lo cual no se recomienda ${ }^{1,9,10}$.

El uso de esteroides tópicos, como complemento de 


\section{"El tratamiento con emolientes (vaselina), previa fototerapia con UVB, puede acelerar la remisión de las lesiones, especialmente, las placas muy gruesas de psoriasis".}

la fototerapia en áreas inaccesibles, como los pliegues y el cuero cabelludo, parece ser apropiado ${ }^{11}$.

\section{Radiación UVB más emolientes}

Los emolientes incrementan la transmisión de la radiación ultravioleta, alterando las propiedades ópticas de las lesiones psoriásicas en la piel y mejorando la eficacia terapéutica. Se recomienda el uso concomitante de emolientes y fototerapia con radiación UVB en el tratamiento de la psoriasis.

El tratamiento con emolientes (vaselina), previa fototerapia con UVB, puede acelerar la remisión de las lesiones, especialmente, las placas muy gruesas de psoriasis ${ }^{12}$.

\section{Radiación UVB más antralina}

La terapia de corto contacto (20 a 30 minutos) con antralina ha mostrado pequeños beneficios adicionales, cuando se administra conjuntamente con rayos UVB. $\mathrm{Su}$ uso ha declinado por la disponibilidad de tratamientos con mejores resultados cosméticos ${ }^{13}$.

\section{Radiación UVB más alquitrán de hulla}

El alquitrán de hulla es un producto de la destilación del carbón, usado desde tiempos ancestrales como tratamiento de varias dermatosis, incluida la psoriasis. El mecanismo de acción no está todavía claramente entendido; se cree que suprime la síntesis de
ADN al disminuir el índice mitótico de los queratinocitos. Su uso se ha visto disminuido puesto que es mal tolerado, puede dejar pigmentación residual en la piel, manchar la ropa y tiene un olor desagradable.

El esquema modificado de Goeckerman es la combinación de alquitrán de hulla crudo más radiación UVB. Se ha descrito en pacientes con psoriasis grave en tratamiento con fototerapia UVB de banda angosta (Narrow Band UVB, NB-UVB), que la terapia ambulatoria con alquitrán de hulla es segura, conveniente, costo-efectiva y puede producir mejoría más rápida de las lesiones, en comparación con la fototerapia sola ${ }^{14}$, sin incrementar la incidencia de cáncer no melanoma ${ }^{13}$.

\section{Radiación UVB más psoralenos tópicos}

La combinación entre psoralenos tópicos y radiación UVB de banda estrecha está menos bien establecida que con la UVA. En el estudio de Jain, et al., se evidenció que el uso de 8-metopsaleno tópico al o,1 \% administrado 15 minutos antes de cada sesión, en un tratamiento de 12 a 24 semanas de duración, mostró una disminución en el número de sesiones de fototerapia, así como la dosis acumulada de NB-UVB. Sin embargo, los resultados no fueron estadísticamente significativos, por lo que se necesitan estudios prospectivos con tamaño mayor de muestra, para delimitar claramente la magnitud de los efectos de la NBUVB en combinación con psoraleno tópico.

Podría ser un recurso para aquellos pacientes resistentes al tratamiento con NB-UVB en monoterapia ${ }^{15}$. 


\section{Radiación UVB e inhibidores de la calcineurina}

La información disponible es extrapolada del tratamiento de pacientes con dermatitis atópica. En varios estudios en animales se ha demostrado que el uso concomitante de inhibidores de la calcineurina y radiación ultravioleta, puede incrementar el riesgo de tumores epiteliales. Sin embargo, no hay datos clínicos sobre el desarrollo de carcinogénesis en humanos. Por lo anterior, se recomienda el uso prudente en casos de psoriasis ${ }^{5-16}$.

\section{PUVA más análogos de la vitamina $D$}

Los análogos de la vitamina D actúan en la psoriasis al unirse a los receptores de vitamina D en los queratinocitos y deteniendo su proliferación.

En varios estudios se ha demostrado que agregar calcipotriol en ungüento a la fototerapia PUVA, resulta en mejoría de las lesiones, con una dosis acumulativa de UVA menor y un inicio más rápido de la reacción terapéutica ${ }^{17}$.

\section{Radiación UVB más análogos de la vitamina $D$}

Hay controversia sobre si el combinarla con vitamina D mejora la eficacia de la radiación UVB (12). En un estudio, la combinación de calcipotriol con radiación UVB fue superior al calcipotriol como monoterapia, aumentando en $34 \%$ la probabilidad de remisión de las lesiones de psoriasis ${ }^{18}$.

Sin embargo, en otro estudio en el cual se comparó la combinación de análogos de vitamina D más UVB con la radiación UVB sola, la terapia combinada no aumentó la probabilidad de remisión de las lesiones de psoriasis y no se encontró una diferencia significativa en la mejoría del puntaje PASI ${ }^{18}$.

Por otro lado, hay otros estudios, incluyendo un metaanálisis, en los que no se ha encontrado ningún beneficio adicional con esta combinación, comparada con la radiación UVB sola ${ }^{19,20}$.

El calcipotriol debe aplicarse con ciertas precauciones para evitar que sea alterado o inhibido por la fototerapia. Si se aplica inmediatamente antes de la fototerapia, esta puede inactivar la molécula. Por esta razón, se recomienda hacer primero la fototerapia y aplicar el calcipotriol al día siguiente día o, al menos, hacerlo dos horas antes de la fototerapia ${ }^{1}$. Aunque hay otros estudios que niegan que sea inactivado por la exposición a la radiación $U^{21}$.

El efecto secundario más común es la dermatitis de contacto irritativa en cara y pliegues (15\%). La hipercalcemia es muy rara, sin embargo, no se debe exceder la dosis de $100 \mathrm{~g}$ a la semana para evitar efectos sobre el metabolismo de calcio ${ }^{22}$.

\section{Radiación UVA más retinoides tópicos}

El tratamiento combinado de retinoides tópicos con UVA, puede mejorar la eficacia terapéutica, con reducción del número de sesiones requeridas y menor dosis acumulada de radiación $\mathrm{UVA}^{23}$.

El tacalcitol o el tazaroteno, ambos con eficacia comparable, mejoran el resultado terapéutico de la fotoquimioterapia con PUVA, en pacientes con psoriasis crónica en placas. Acelera la reacción al tratamiento, con lo cual se requiere una menor dosis acumulada de UVA $\mathrm{y}$, por lo tanto, se reducen los efectos adversos a largo plazo de la radiación $\mathrm{UVA}^{24}$.

\section{Radiación UVB más retinoides tópicos}

$\mathrm{Al}$ agregar tazaroteno a la fototerapia UVB, se mejora y acelera la eficacia, manteniendo aceptables la seguridad y la tolerabilidad. Produce mejoría de la descamación, elevación de la placa y eritema durante todo el tratamiento, con una dosis media acumulativa de UVB significativamente menor. La dosis necesaria para alcanzar el PASI 50 se disminuye en 50 \% o más, con la adición del tazaroteno ${ }^{25}$.

Este tratamiento combinado puede causar aumento de la sensibilidad a las quemaduras, por lo cual en varios estudios se recomienda disminuir un tercio de la dosis de la UVB y aumentarla según la tolerancia ${ }^{6,10,26}$.

Los efectos secundarios, como quemaduras, descamación y dermatitis irritativa, asociados con los retinoides, limitan la aceptación de este tratamiento por los pacientes ${ }^{1}$. Estos efectos pueden reducirse si se adicionan esteroides tópicos al tratamiento ${ }^{27}$. 


\section{Radiación UVB más retinoides tópicos Vs. radiación UVB más análogos de la vitamina D3}

En un estudio piloto con 10 pacientes, se comparó la radiación UVB más tazaroteno con la UVB más calcipotriol, cada combinación en la mitad del cuerpo de un mismo individuo, y se encontró que no había diferencias significativas entre ambos esquemas. Con ambos se disminuyó la dosis acumulada de UVB. Se consideró que cualquiera de estos dos esquemas pueden considerase como opción de primera línea en las psoriasis moderadas a graves ${ }^{28}$.

\section{Radiación UVB más análogos de la vitamina $\mathrm{D}$ y esteroides}

No hay estudios controlados de asignación aleatoria sobre este esquema. En un reporte de ocho casos, se administró Dovobet ${ }^{\circledR}$ (50 $\mu \mathrm{g}$ de monohidrato de calcipotriol más 0,5 mg de dipropionato de betametasona) en ungüento más fototerapia UVB; esta combinación fue extremadamente efectiva y se consideró como tratamiento de primera línea en las psoriasis moderadas a graves. Además, el Dovobet ${ }^{\circledR}$ actuó como ahorrador de esteroides y disminuyó el número requerido de sesiones de fototerapia ${ }^{29,30}$.

\section{Fototerapia más retinoides orales}

La combinación de fototerapia y retinoides orales se conoce como RePUVA (Retinoid PUVA) o ReUVB (Retinoid $U V B)$. Los retinoides orales son un subgrupo de análogos de la vitamina A, naturales o sintéticos. Desde los años 70 se han usado en monoterapia o combinados con fototerapia, ya que modulan las tres características principales de la psoriasis: la diferenciación y la hiperproliferación de queratinocitos, y la infiltración del tejido por linfocitos T. Sin embargo, el mecanismo exacto por el cual los retinoides interfieren con estos eventos no se ha establecido ${ }^{4}$.

Tres retinoides sintéticos se han comercializado para uso sistémico ${ }^{30}$ : el etretinato (éster aromático), un profármaco que es convertido en acitretín; el acitretín, ácido libre derivado del etretinato, y la isotretinoína (13-cis-ácido retinoico).

La primera publicación sobre el uso de etretinato
(Tigason $^{\circledR}$ ) en el tratamiento de la psoriasis se hizo en 1975. No obstante, y a pesar de su eficacia, su uso fue limitado por su desfavorable perfil farmacocinético, ya que este medicamento tiene una vida media aproximada de 120 días y es muy lipofílico; se almacena en el tejido graso y su eliminación es prolongada: se requieren aproximadamente dos años para completarla, después de la suspensión del tratamiento. Por esta razón, en marzo de 1998 el etretinato fue retirado del mercado de Estados Unidos y fue reemplazado por el acitretín (Neotigason ${ }^{\circledR}$ ), el cual es menos lipofílico y tiene una vida media aproximada de dos días. Sin embargo, la ingestión concomitante de alcohol y acitretín lleva a la transesterificación del acitretín a etretinato; se desconoce la cantidad exacta de alcohol necesaria para producir este efecto. El acitretín se debe administrar con las comidas, lo que potencia su absorción y mejora la biodisponibildad de dos a cinco veces. Entre los retinoides, la isotretinoína es el que menos efecto tiene en esta enfermedad ${ }^{4 \cdot 31}$.

En 1996, la Food and Drug Administration (FDA) aprobó los retinoides sistémicos para el tratamiento de la psoriasis grave $\mathrm{e}^{32,33}$.

Un avance importante para el tratamiento de la psoriasis, ha sido la combinación de la fototerapia con etretinato o acitretín. Se han llevado a cabo varios ensayos multicéntricos de asignación aleatoria para determinar la eficacia de estos medicamentos como monoterapia o combinados con fototerapia (PUVA o UVB), para el tratamiento de la psoriasis; el tratamiento combinado produjo una remisión más rápida, con menos radiación y retinoides, con mayor comodidad para el paciente y menores $\operatorname{costos}^{31-34}$.

Sin embargo, la monoterapia con retinoides no produce una remisión completa, como sí lo logran los tratamientos combinados; en un sinnúmero de estudios clínicos, se sugiere que estos últimos pueden aumentar la eficacia y la tolerabilidad e, inclusive, pueden disminuir los efectos secundarios al aumentar su perfil de seguridad ${ }^{18}$.

Esto lo informaron Anstey y Hawk, quienes observaron una reducción del $30 \mathrm{al} 40 \%$ en el número de tratamientos y en las dosis acumuladas de UVA, en cuatro mujeres con psoriasis, en comparación con las mismas pacientes cuando no se utilizaron retinoides ${ }^{35}$.

\section{Tratamiento combinado con retinoides}

Los estudios en que se utiliza el acitretín en combinación con fototerapia (PUVA/UVB) han demostrado 
la clara superioridad del tratamiento combinado con estos dos agentes sobre cada uno de ellos como monoterapia, en casos de psoriasis en placas e, inclusive, en la forma pustulosa $a^{3,31,36}$.

Las principales ventajas de la combinación fueron: aceleración de la tasa de remisión de las lesiones; mejoría de las lesiones en casos de falla terapéutica de la monoterapia con retinoides o con fototerapia; $y$, disminución del número de las sesiones, con menor riesgo de efectos cancerígenos propios de la radiación, los cuales dependen claramente de la dosis ${ }^{30,31,36}$. Además, los retinoides orales han demostrado proteger contra el cáncer de piel ${ }^{32}$.

Como los retinoides pueden producir adelgazamiento del estrato córneo, la dosis inicial de fototerapia (PUVA o UVB) es el 50 \% de la habitual y sus incrementos son graduales. El acitretín se inicia una a dos semanas antes de la fototerapia, a dosis de 10 a $25 \mathrm{mg}$ diarios $^{31}$.

\section{RePUVA}

En varios estudios se ha evaluado la asociación de retinoides con PUVA (RePUVA), comparándola con placebo o con otros esquemas, encontrándose que el RePUVA es más eficaz que la terapia PUVA sola, en la remisión de la psoriasis en placas; además, requiere dosis menores de retinoide y de PUVA, en comparación con la monoterapia ${ }^{3}$.

En un estudio que apoya esto, la dosis media acumulada de UVA fue $42 \%$ menor en el grupo con acitretín más PUVA que en el grupo de placebo más PUVA ${ }^{30}$.

En un estudio de asignación aleatoria y doble ciego, se evaluaron 60 pacientes con psoriasis grave, cuyo tratamiento con acitretín más PUVA resultó en remisión de los síntomas en el $96 \%$ de los casos, en comparación con la disminución en el 80 \% con la PUVA sola ${ }^{33}$. En otro estudio de características similares, se comparó el tratamiento con placebo más PUVA $(n=25)$ con el de acitretín más PUVA ( $n=23)$, y se demostró que aproximadamente el $80 \%$ de los pacientes que recibieron placebo más PUVA estaban limpios de lesiones o casi limpios, en comparación con el 96 \% en el grupo de RePUVA 4 .

También, se han publicado estudios sobre isotretinoína y PUVA, como uno controlado y de asignación aleatoria en 60 pacientes, en el cual se estableció que la combinación de PUVA más etretinato y la de PUVA más isotretinoína fueron superiores a la monoterapia con PUVA. Los autores recomiendan la terapia con isotretinoína en mujeres en edad fértil, en las cuales el etretinato está contraindicado, aunque la eficacia de este último es un poco mayor que la de la isotretinoína ${ }^{35}$.

Una alternativa a la terapia PUVA oral es el baño de
PUVA (baño de psoraleno más UVA), el cual también se puede combinar con retinoides y permite reducir la dosis efectiva de los rayos UVA, con buena respuesta terapéutica ${ }^{30}$. Con esta modalidad no se presentan los efectos gastrointestinales que produce el psoraleno oral y, además, hay una recuperación más rápida de la fototoxicidad y menor potencial carcinogénico ${ }^{4}$.

En un análisis combinado de dos cohortes de 944 pacientes suecos y finlandeses con psoriasis y tratados con baño de PUVA, no se encontró aumento en el riesgo de carcinoma espinocelular, después de una media de seguimiento de 14,7 años ${ }^{4}$.

En un estudio pequeño, cuatro pacientes con psoriasis grave fueron tratados con acitretín (o,5 mg/ kg/ día) y baño de PUVA, tres a cinco veces a la semana, durante cuatro semanas. Todos lograron una tasa de éxito superior al $90 \%$ al final del estudio y no se presentaron recaídas después de tres meses ${ }^{4}$.

\section{ReUVB}

La fototerapia con UVB de banda angosta (NB-UVB) como monoterapia, es muy eficaz contra la psoriasis en placas, pero se requieren múltiples sesiones de fototerapia, lo cual impide un buen cumplimiento del tratamiento por parte del paciente.

Por otra parte, el acitretín es moderadamente eficaz como monoterapia, pero, al combinarlo con radiación UVB, se disminuye el número de sesiones necesarias para lograr la mejoría clínica de lesiones que fueron resistentes a otras modalidades terapéuticas $(31,33)$. Como se demostró en un estudio de 40 pacientes con psoriasis en placas resistente a los tratamientos tópicos con esteroides de alta potencia, como tazaroteno y calcipotriol, la combinación de dosis bajas de acitretín (25 mg/día) más NB-UVB, tres veces por semana, resultó en una mejoría de más de $75 \%$ en 29 $(72,5 \%)$ pacientes, mientras que solo $5(12,5 \%)$ obtuvieron menos del 50\% de mejoría ${ }^{31}$.

En dos estudios controlados y de asignación aleatoria y en un estudio abierto, se evaluó la asociación de acitretín y etretinato con UVB, comparada con UVB como monoterapia, y se demostró que la ReUVB es más eficaz que la UVB sola para obtener remisión de la psoriasis en placas ${ }^{3}$.

En otro estudio de 82 pacientes con psoriasis grave, se comparó el acitretín más UVB con el placebo más UVB, y se demostró reducción del PASI en $79 \%$ en el grupo con ReUVB, comparada con una de $35 \%$ en el grupo con placebo ${ }^{33}$.

En un estudio de Ruzicka, et al., 40 pacientes se sometieron al tratamiento con acitretín más UVB y se 


\section{"La fototerapia con UVB de banda angosta (NB-UVB) como monoterapia, es muy eficaz contra la psoriasis en placas, pero se requieren múltiples sesiones de fototerapia, lo cual impide un buen cumplimiento del tratamiento por parte del paciente".}

compararon con 38 pacientes que recibieron UVB más placebo; se demostró que el $60 \%$ del primer grupo presentó disminución del PASI, en comparación con el $24 \%$ en el grupo con placebo más UVB ${ }^{1}$.

En pacientes con psoriasis vulgar persistente, el tratamiento combinado de acitretín en dosis de $50 \mathrm{mg} /$ día más UVB, resultó en un alto grado de remisión de las lesiones, con menos sesiones de radiación que con la fototerapia sola ${ }^{33}$.

La combinación de etretinato o acitretín con UVB, ha demostrado ser más eficaz en la psoriasis que la monoterapia con retinoide o con UVB; los resultados son variables con la isotretinoína ${ }^{30,35}$.

Parece que la isotretinoína es un poco menos efectiva que el etretinato en el tratamiento de la psoriasis, cuando se utiliza como monoterapia. Sin embargo, cuando la isotretinoína se combina con fototerapia, su eficacia es comparable a la de la fototerapia más etretinato. La isotretinoína más la NB-UVB acelera significativamente la reacción terapéutica en la psoriasis vulgar y se reduce la dosis acumulativa en comparación con la NB-UVB más placebo; por lo tanto, la isotretinoína puede considerarse un agente coadyuvante a la fototerapia NB-UVB en lugar del etretinato o el acitretín, especialmente, en mujeres en edad fértil ${ }^{35}$.

En conclusión, la combinación de NB-UVB y acitretín es un tratamiento muy eficaz y bien tolerado, incluso en pacientes con psoriasis persistente, y mejora significativamente las lesiones con menos efectos secundarios que la monoterapia ${ }^{31}$.

\section{RePUVA versus ReUVB}

Finalmente, son pocos los estudios publicados en los cuales se compara la efectividad de la RePUVA con la de la ReUVB.

En un estudio de asignación aleatoria publicado en 2008, se comparó el acitretín más NB-UVB con el acitretín más PUVA, tres veces a la semana, en 6o pacientes con psoriasis en placas de moderada a grave. Se logró remisión de la psoriasis en $56,6 \%$ de los pacientes tratados con acitretín más NB-UVB y en 63,3\% de aquellos tratados con acitretín más PUVA. Todos estos pacientes permanecieron libres de lesiones tres meses después de finalizar el tratamiento. En conclusión, el tratamiento con acitretín más NB-UVB es eficaz y bien tolerado en la psoriasis moderada a grave en placas, con un efecto terapéutico equivalente al del acitretín más PUVA ${ }^{15}$.

A pesar de que en este y otros estudios se demuestra una ligera superioridad del RePUVA sobre el ReUVB, los efectos secundarios de la PUVA, como náuseas, necesidad de protección contra la luz solar y aumento del riesgo de cáncer de piel por el uso de psoralenos, son algunos de los aspectos negativos que afectan el cumplimiento y la tolerancia del tratamiento en $\mathrm{mu}$ chos pacientes. Aunque los efectos secundarios del tratamiento con NB-UVB a largo plazo no están tan bien establecidos, como sí lo están con el PUVA, el ReUVB puede ser una buena alternativa terapéutica, en pacientes con psoriasis que normalmente se trataban con RePUVA. 
La NB-UVB sola o en combinación con un retinoide oral (acitretín) es el tratamiento de primera línea para pacientes con psoriasis extensa. Esta combinación ha demostrado acelerar la mejoría de las lesiones y permitir un periodo más prolongado de remisión ${ }^{1}$.

\section{Indicaciones para RePUVA o ReUVB}

En múltiples estudios se ha confirmado la eficacia del acitretín en el tratamiento de las diferentes formas de psoriasis. La psoriasis pustulosa y la eritrodérmica son más sensibles al acitretín como monoterapia que la psoriasis en placas, la cual mejora pero más lentamente.

Aunque el acitretín es menos eficaz como monoterapia para la psoriasis en placas, su combinación con otros agentes, especialmente UVB o PUVA, puede mejorar la eficacia ${ }^{3,4}$.

La combinación de retinoides más fototerapia debe considerarse en pacientes con psoriasis en placas, de moderada a grave, que no haya mejorado con monoterapia de UVB, de PUVA o acitretín, en aquellos con acceso limitado a la fototerapia o en quienes el metotrexato o la ciclosporina hayan sido ineficaces o hayan producido efectos secundarios intolerables. La ReUVB es preferible a la RePUVA debido a los riesgos documentados de carcinoma escamocelular con esta última terapia ${ }^{1}$.

Debido a que con frecuencia el acitretín no es bien tolerado, generalmente se usa a dosis bajas (10 a 25 $\mathrm{mg} /$ día), como parte de la terapia combinada ${ }^{4}$. Se debe tomar una vez al día y con las comidas, con leche o ambas, para mejorar su absorción ${ }^{36}$. La dosis no debe superar los $25 \mathrm{mg}$ diarios, con el fin de limitar la incidencia y la gravedad de los efectos secundarios, ya que el acitretín, en dosis entre 25 y $50 \mathrm{mg}$ diarios, se asocia con una incidencia mayor de estos eventos ${ }^{3,4}$.

El acitretín se puede empezar dos semanas antes o al mismo tiempo que la fototerapia ${ }^{1,4}$. En este último caso, la dosis de radiación debe disminuirse en el $50 \%$ una semana antes de iniciar el medicamento, para evitar una reacción de fotosensibilidad ${ }^{2,4}$.

La fototerapia se inicia con el 30 al $50 \%$ de la dosis mínima de eritema o según el fototipo; se realiza tres veces por semana, con aumentos semanales de $10 \%$ de la dosis mínima de eritema en las primeras 20 sesiones y, una vez lograda la remisión, se suspende el acitretín y se continúa con fototerapia ${ }^{1,2}$.

El aumento en la dosis de radiación UV deben ser más gradual y hacerse con mayor cautela en los pacientes que reciben retinoides que en aquellos que no los reciben, debido a un mayor riesgo de eritema inducido por la radiación ${ }^{4}$.

La monoterapia con acitretín o con etretinato pro- ducen los mejores resultados en la psoriasis pustulosa palmo-plantar (localizada) o la generalizada (von Zumbusch), y se considera que es la primera elección para este tipo de psoriasis, a pesar de que sólo unos pocos estudios con un número pequeño de pacientes apoyan esta recomendación. También, es una opción de tratamiento en combinación con la fototerapia para la psoriasis en placas de moderada a grave $\mathrm{3}^{3,30,37}$.

La principal ventaja del acitretín sobre otros agentes sistémicos (como metotrexato, ciclosporina o productos biológicos) es que no tiene efecto inmunosupresor; por lo tanto, está indicado en pacientes con psoriasis y con antecedente de infección por HIV, hepatitis B o hepatitis C, o de neoplasia maligna, en quienes están contraindicados los inmunosupresoros sistémicos ${ }^{4,32,34}$.

La RePUVA y la ReUVB están contraindicadas en el embarazo y la lactancia, en mujeres en edad fértil cuando existe otra alternativa adecuada, en las dermatosis asociadas que se exacerban con la radiación UV, en la insuficiencia hepática (más de dos veces el valor normal de las enzimas), en la hepatitis y en la insuficiencia renal de moderada a grave ${ }^{30,36}$. No se recomienda en niños, ya que hay informes de cambios óseos. Si los beneficios superan significativamente los riesgos, se inicia el tratamiento y se debe estar atento a cualquier anormalidad en los parámetros de crecimiento, diligenciando tablas de crecimiento.

El acitretín y el etretinato no se deben administrar en pacientes con alteraciones graves de la retina, pero, si es mayor el beneficio que el riesgo, el paciente debe ser evaluado por un oftalmólogo, quien decidirá si administrar o no el medicamento.

\section{Fototerapia más metotrexato oral}

El metotrexato fue implementado como tratamiento efectivo en la psoriasis en la década de los 70, y aún ahora es muy usado. Es un análogo estructural del ácido fólico e inhibe reversiblemente la dihidro-folatorreductasa, enzima requerida para la reducción del ácido fólico a ácido tetrahidrofólico. Por medio de este mecanismo, el metotrexato previene la síntesis de ADN y, en la psoriasis, disminuye la tasa de replicación celular.

Tiene múltiples efectos secundarios que pueden ser benignos o, incluso, amenazar la vida. Los efectos secundarios más comunes son: náuseas, vómito, anorexia, anemia macrocítica y fototoxicidad. La anemia macrocítica se previene usando 1 a $5 \mathrm{mg}$ diarios de ácido fólico por vía oral. Además, puede causar convulsiones, hepatotoxicidad, falla renal, supresión de la médula ósea, fibrosis pulmonar y alteraciones linfoproliferativas. La hepatotoxicidad es el efecto secun- 
dario más serio a largo plazo; por lo tanto, los pacientes deben ser controlados periódicamente con pruebas de función hepática. Está contraindicado en enfermedad renal o hepática, en el embarazo (es un medicamento de clase X, por lo tanto, es teratogénico), en la depresión de la médula ósea y en personas con bajo coeficiente intelectual ya que puede ser de difícil comprensión su administración semanal. Es importante advertir al paciente que no debe ingerir alcohol ${ }^{6,38}$.

El metotrexato se puede combinar con PUVA, con UVB y con etretinato. El objetivo de dichas combinaciones es disminuir la dosis y la toxicidad subsecuente; sin embargo, existe preocupación en cuanto a la inducción de cáncer en el tratamiento combinado de metotrexato y fototerapia.

El metotrexato también se ha combinado exitosamente con ciclosporina en pacientes con psoriasis grave persistente, la mayoría de los cuales tenían artropatía psoriásica, aunque sobre esta combinación no hay estudios a largo plazo ${ }^{39}$.

En seis estudios se examinó el efecto de la combinación del metotrexato más fototerapia y se concluyó que se alcanza un efecto aditivo cuando se combina la fototerapia con el metotrexato ${ }^{40,41}$.

\section{PUVA más metotrexato}

Aunque se ha sugerido que el metotrexato combinado con PUVA puede predisponer a los pacientes a desarrollar carcinoma escamocelular, esta combinación ha sido efectiva en casos de psoriasis resistente a otros medicamentos. Tiene la ventaja de que puede disminuir la radiación UVA total necesaria para alcanzar la remisión, así como disminuir la radiación requerida para el tratamiento de mantenimiento ${ }^{10}$.

Puede ser una combinación útil en la fase de remisión en pacientes con psoriasis persistente, aunque no se emplea muy frecuentemente pues se considera que aumenta el riesgo de carcinoma escamocelular y, además, porque el metotrexato y la radiación UVA tienen un efecto aditivo inmunosupresor.

El metotrexato se debe iniciar tres semanas antes de la PUVA para lograr que las placas de psoriasis se adelgacen; además, se debe disminuir la dosis de radiación ultravioleta A para evitar reacciones fototóxicas.

Es importante evitar la ingestión del metotrexato concomitantemente con psoralenos, para disminuir el riesgo de hepatotoxicidad. A las dos semanas de haber iniciado el tratamiento combinado, se debe disminuir la dosis del metotrexato a la mitad y se continúan las sesiones de PUVA tres veces a la semana hasta que se alcance una remisión del $95 \%$. Si a las dos semanas de iniciada la terapia combinada no hay mejoría clínica, se debe continuar con iguales dosis de metotrexato y aumentar la dosis de radiación UVA, hasta conseguir la remisión. Esta combinación se debe mantener durante dos meses hasta que se induzca la fase de remisión de la enfermedad, después de lo cual se suspende el metotrexato ${ }^{2,40,41}$.

Morison, et al., estudiaron 30 pacientes a quienes suministraron un esquema para remisión de $15 \mathrm{mg}$ de metotrexato a la semana, durante tres semanas, seguido de un tratamiento convencional de metotrexato más PUVA y, por último, disminuyeron gradualmente la PUVA durante la fase de remisión. Hubo remisión en 28 de 30 (93\%) pacientes, incluidos cinco de los seis que habían sido previamente resistentes a la monoterapia con PUVA. La principal desventaja de este esquema fue el efecto aditivo en la carcinogénesis, especialmente, del carcinoma escamocelular. A los cuatro meses del tratamiento, dos tercios de los pacientes tuvieron recaída de la enfermedad ${ }^{7,10}$.

En un estudio de asignación aleatoria, Shehzad, et al., encontraron que los pacientes que recibieron PUVA en combinación con metotrexato alcanzaron la remisión más temprano que aquellos tratados con metotrexato o con PUVA en monoterapia; no obstante, hay que tener en cuenta que el riesgo de cáncer a largo plazo puede ser mayor ${ }^{40,41}$.

En conclusión, aunque el metotrexato se puede combinar con PUVA, esta asociación no se recomienda en la actualidad pues aumenta el riesgo de carcinoma escamocelular ${ }^{1}$.

\section{NB-UVB más metotrexato}

Desde su introducción en 1988, la UVB de banda estrecha ha mostrado buena eficacia en el tratamiento de la psoriasis, usada con una variedad de medicamentos tópicos y sistémicos.

La combinación de metotrexato con NB-UVB parece ser sinérgica: la reacción ocurre con menos dosis acumulada de metotrexato y de UVB. La desventaja de este esquema es que la UVB puede producir remisión transitoria, ya que al suspender el metrotexato se puede producir un rebote ${ }^{2,10}$.

En los pocos estudios en que se han combinado el metotrexato y la NB-UVB, se encontró que esta mezcla es capaz de lograr remisión de la enfermedad en menos tiempo y con menos dosis acumulada de $\mathrm{UVB}^{42,43}$.

Asawanonda, et al., en un estudio de asignación aleatoria, estudiaron 24 pacientes con psoriasis en placas que cubrían al menos el $20 \%$ de la superficie corporal, los cuales recibieron $15 \mathrm{mg}$ de metotrexato a la semana 
o placebo, durante tres semanas, antes de iniciar la fototerapia con NB-UVB. Los pacientes fueron tratados con el agente oral más fototerapia durante 24 semanas, o hasta que el PASI disminuyera a menos del $10 \%$ del puntaje original. Encontraron que los que recibieron terapia combinada alcanzaron el PASI requerido en una mediana de cuatro semanas, tiempo significantemente menor que en el grupo con placebo más NB-UVB (90 \% Vs. 38,5\%), por lo cual requirieron menos sesiones de fototerapia y, por consiguiente menor dosis acumulada de NB-UVB que cuando esta se usó como monoterapia, además, con dosis acumuladas de metotrexato más bajas, lo que disminuye el riesgo de hepatotoxicidad. Una limitación de este estudio fue la falta de un grupo con metotrexato en monoterapia ${ }^{1,43,13}$.

Mahajan, et al., encontraron que, cuando se combina metotrexato más NB-UVB, hay una remisión más rápida de las lesiones por su acción sinérgica, debido a que ambos tienen una acción antimitótica, antiproliferativa y antiinflamatoria, y además, este medicamento produce un adelgazamiento de las placas de psoriasis, por lo cual ayuda a una penetración más profunda de la UVB en la dermis ${ }^{42}$.

\section{Fototerapia más terapia biológica}

En la actualidad, el tratamiento biológico se ha convertido en una alternativa en la psoriasis, ya que brinda eficacia y mejora la calidad de vida, principalmente, en pacientes que no han mejorado de forma adecuada con otros tratamientos tópicos o sistémicos ${ }^{44,45}$.

La asociación de productos biológicos con fototerapia, principalmente NB-UVB, ha sido un paso más en los avances del tratamiento de la psoriasis; brinda una resolución más rápida y completa de las lesiones y logra disminuir la dosis de radiación necesaria la remisión de las lesiones ${ }^{1}$.

Una gran ventaja de la terapia biológica y por lo cual se ha incrementado su uso en los últimos años, es su perfil de eficacia y seguridad, con menor toxicidad sistémica ${ }^{44}$.

Los medicamentos biológicos se clasifican, según su origen, en:

Inhibidores del factor de necrosis tumoral alfa: se denominan de esta manera por la forma de actuar directa o indirectamente sobre el factor de necrosis tumoral (TNFa), ejerciendo un papel en el proceso inflamatorio de la psoriasis. Este grupo puede subclasificarse, según el tipo de molécula que actúa, en:
- Anticuerpos monoclonales. Pueden ser quiméricos, como el infliximab, humanizados como el siplizumab (que no se encuentra disponible en la actualidad), y humanos, como el adalimumab.

- Proteínas de fusión. Combinan porciones de proteínas y son similares a los anticuerpos. Estas moléculas, por ser derivadas de productos humanos, tienen poca capacidad inmunogénica. El etanercept es la proteína de fusión más usada actualmente en la psoriasis.

Inhibidores de interleucinas (IL), IL-12 e IL-23: El ustekinumab, un anticuerpo monoclonal humano, es el modelo inicial. Este ha emergido con el descubrimiento de las células Th17 como unas de las principales efectoras en la psoriasis, ya que el ustekinumab se une a la subunidad p40 de las IL-12 e IL-23, y así, evita la generación de células Th1 y Th17, lo cual modifica los efectos inflamatorios de estas citocinas ${ }^{44}$.

Inhibidores de IL-17:: Es el producto biológico introducido más recientemente. Se trata de un anticuerpo monoclonal humano recombinante, que se une selectivamente a la IL-17 $\mathrm{A}^{46}$. Actualmente, el secukinumab $\left(\right.$ Cosentyx $\left.{ }^{\circledR}\right)$ se encuentra aprobado para el tratamiento de la psoriasis.

En muchos estudios se ha demostrado que, al combinar los productos biológicos con NB-UVB, se aumenta su eficacia, como sucede con el etanercept, el adalimumab y el alefacept. Sin embargo, por el incremento en el riesgo de efectos secundarios, incluidas la carcinogénesis asociada a la fototerapia y las reacciones fototóxicas sistémicas, sólo se recomienda su uso en pacientes que han sido intolerantes a las altas dosis de fototerapia o a los demás medicamentos ${ }^{44}$.

La combinación de productos biológicos con fototerapia aumenta la eficacia, produce una remisión más rápida, tiene una adecuada tolerancia y es una excelente opción en pacientes que recaen o no mejoran (grado de recomendación $\mathrm{B}$, nivel de evidencia 2$)^{47}$.

\section{Adalimumab y fototerapia}

El adalimumab es un anticuerpo monoclonal anti-TNF $\alpha$ efectivo para el tratamiento de la psoriasis moderada a grave. Su eficacia está demostrada por numerosos estudios en los que se ha logrado un PASI 75 (grado de recomendación $\mathrm{A}$, nivel de evidencia 1$)^{44}$.

En un estudio en 2011, por Wolff, et al., se comprobó la eficacia de la combinación de fototerapia NB-UVB y adalimumab. Se evaluaron cuatro pacientes con psoriasis moderada a grave y se encontró una mejoría más rápida de las lesiones, después de seis semanas de tra- 
tamiento. En cada paciente se eligió al azar una mitad del cuerpo para recibir terapia combinada y, la otra, solo adalimumab; la media del PASI fue 2 en las áreas que recibieron radiación NB-UVB frente a una media de 6,9 en las que se utilizó la monoterapia, lo cual correspondió a una reducción media global del PASI de $86 \%$ Vs. $53 \%$ con respecto al valor basal. En la semana 12, el PASI fue de 4,5 en las área irradiadas Vs. 7,3 en las no irradiadas (diferencia no significativa), lo que sugiere que para obtener una reacción terapéutica adecuada puede ser necesario un tratamiento combinado a largo plazo, lo cual conlleva mayores efectos secundarios, por lo que en la actualidad conviene usar este tratamiento con precaución ${ }^{48}$.

Los principales efectos secundarios del adalimumab son: reacciones en el sitio de aplicación, generalmente leves, y tuberculosis, que generalmente se manifiesta en el primer año de tratamiento y refleja una enfermedad latente; además, hay reportes de casos de lupus eritematoso sistémico inducido por adalimumab que remiten al suspender el tratamiento ${ }^{49}$.

No hay estudios sobre este tratamiento en pacientes con falla renal, falla hepática o ambas ${ }^{45}$. Recientemente, su uso fue aprobado en niños, sin haber demostrado más efectos adversos que en la población general ${ }^{50}$.

En estudios recientes se ha confirmado que la combinación de adalimumab más fototerapia acelera y hace significativamente más rápida la remisión de las placas de psoriasis, en comparación con la monoterapia. Bagel, et al., estudiaron 20 pacientes con psoriasis moderada a grave, a quienes se les suministró, semanalmente, 40 mg de adalimumab por vía subcutánea y tres sesiones de fototerapia NB-UVB, durante 12 semanas, con un seguimiento de 12 semanas al finalizar el tratamiento; se encontró un PASI 75 en el $50 \%$ de los pacientes a la semana 4 y, a la semana 12 , uno de $95 \%$, sin evidencia de recaídas al finalizar el estudio (semana 24) ${ }^{49,51}$.

\section{Alefacept y fototerapia}

El alefacept es una proteína de fusión dimérica LFA3/IgG1 que bloquea la interacción entre la célula presentadora de antígeno y la célula T, al unirse a la molécula CD2 del linfocito e inducir su apoptosis. El alefacept fue aprobado para el tratamiento de la psoriasis en el $2003 \mathrm{y}$, desde entonces, en diferentes estudios se ha demostrado su efectividad, principalmente a largo plazo, y con remisiones duraderas $^{47,52,54}$. Es importante vigilar la toxicidad hematológica, pues se han documentado linfopenia; se recomienda el seguimiento con hemoleucograma y recuento de $\mathrm{CD} 4 \mathrm{y} \mathrm{CD}^{47}$.

\section{Infliximab y fototerapia}

En la literatura científica revisada, no se encontraron reportes hasta la fecha que documenten la eficacia y la seguridad de la combinación de infliximab con fototerapia.

\section{Etanercept y fototerapia}

Es una proteína recombinante humana del receptor del TNFa, que se une a este y neutraliza su acción, y tiene una baja tasa de inmunogenicidad ( $<2 \%)$. Fue aprobado en 2004 por la FDA para el tratamiento de la psoriasis en placas de moderada a grave y de la artritis psoriásica; la dosis estándar es de $50 \mathrm{mg}$ por vía subcutánea dos veces a la semana durante 12 semanas y, posteriormente, $50 \mathrm{mg}$ una vez a la semana por tiempo indefinido ${ }^{55}$. Este medicamento no requiere ajuste de la dosis en la enfermedad hepática o renal.

El tratamiento combinado de etanercept más fototerapia ha sido objeto de estudio, en los últimos años. Se han publicado múltiples estudios, en los que se demuestra que dicha combinación es más efectiva que la monoterapia para el tratamiento de la psoriasis, incluso, haciendo necesarias menores dosis de etanercept $\mathrm{t}^{45,48,51,56,57}$.

\section{Ustekinumab y fototerapia}

El ustekinumab inhibe la subunidad p40, común a la IL-12 y la IL-23, y de esta forma, modula la generación de células Th1 y Th17, importantes en la patogénesis de la psoriasis $45,57,58$.

Fue aprobado por la FDA en 2009, para la psoriasis moderada a grave (grado de recomendación A y nivel de evidencia 1).

La dosis usada es de $45 \mathrm{mg}$ de ustekinumab por vía subcutánea en la semana o y la 4, y después, $45 \mathrm{mg}$ cada 12 semanas; si el peso corporal es mayor de 100 $\mathrm{kg}$, la dosis es de $90 \mathrm{mg}$. Al suspender el ustekinumab se presentan recaídas a las 15 semanas, por lo que se recomienda mantenimiento continuo $0^{45,46}$.

Con respecto a la combinación de ustekinumab con fototerapia, solo hay un estudio del año 2011, de Wolff, et al., en el que se incluyeron 10 pacientes tratados con ustekinumab a las dosis recomendadas y, a los 2 días, se les determinó la dosis mínima de eritema. Esta se aplicó en un hemicuerpo escogido aleatoriamente, con sesiones tres veces a la semana durante seis semanas. Se evaluó semanalmente la reacción terapéutica con el PASI y se encontró que la NB-UVB la acelera significativamente, con una reducción del PASI de $82 \%$ en el lado irradiado contra $54 \%$ en el no irradiado, a la semana 6 
de tratamiento ${ }^{5}$. Hasta la fecha, no hay estudios adicionales que demuestren la seguridad y la efectividad de la combinación de ustekinumab y fototerapia.

\section{CONCLUSIONES}

Aproximadamente el $80 \%$ de los pacientes con psoriasis tiene una enfermedad leve. Sin embargo, el tratamiento debe ser individualizado de acuerdo con las expectativas, las necesidades, y las características de cada paciente.

La fototerapia se considera una de las modalidades más efectivas para el tratamiento de la psoriasis y puede usarse como monoterapia o como terapia combinada, con medicamentos tópicos, sistémicos o biológicos.

El tratamiento combinado permite alcanzar una remisión más rápida de las lesiones y limitar la aparición de efectos secundarios a la monoterapia prolongada.

El tratamiento de los pacientes con psoriasis debe enfocarse siempre en las necesidaddes físicas y psicológicas de cada paciente, ya que también debe considerarse que, según el impacto de esta enfermedad en la calidad de vida, se deben tomar decisiones importantes en cuanto a las terapias que se usan, teniendo en cuenta también otros aspectos como la gravedad, la localización, la edad del paciente y las enfermedades concomitantes.

\section{REFERENCIAS}

1. Calle J, Rivas AM, Ruiz MC. Manejo de la psoriasis con luz ultravioleta A más psolareno (PUVA) y luz ultravioleta B (UVB). Rev Asoc Col Dermatol. 2009;17:162-71.

2. Sbidian E, Maza A, Montaudié H, Gallini A, Aractingi S, Aubin F, et al. Efficacy and safety of oral retinoids in different psoriasis subtypes: A systematic literature review. J Eur Acad Dermatol Venereol. 2011;25(Suppl.2):28-33.

3. Lee CS, Li K. A review of acitretin for the treatment of psoriasis. Expert Opin Drug Saf. 2009;8:769-79.

4. Lapolla W, Yentzer BA, Bagel J, Halvorson CR, Feldman SR. A review of phototherapy protocols for psoriasis treatment. J Am Acad Dermatol. 2011;64:936-49.

5. Menter A, Korman NJ, Elmets CA, Feldman SR, Gelfand JM, Gordon $\mathrm{KB}$, et al. Guidelines of care for the management of psoriasis and psoriatic arthritis. Section 3. Guidelines of care for the management and treatment of psoriasis with topical therapies. J Am Acad Dermatol. 2009;60:643-59.

6. Aaronson DS, Lebwohl M. Review of therapy of psoriasis: The prebiologic armamentarium. Dermatol Clin. 2004;22:379-88, viii.

7. González C, Londoño A, Gómez L. Guías basadas en la evidencia para el manejo de la psoriasis en Colombia. Bogotá: Panamericana Formas e Impresos; 2012.
8. Morison WL, Parrish JA, Fitzpatrick TB. Controlled study of PUVA and adjunctive topical therapy in the management of psoriasis. Br J Dermatol. 1978;98:125-32.

9. Schmoll M, Henseler T, Christophers E. Evaluation of PUVA, topical corticosteroids and the combination of both in the treatment of psoriasis. Br J Dermatol. 1978;99:693-702.

10. Meola T, Soter NA, Lim HW. Are topical corticosteroids useful adjunctive therapy for the treatment of psoriasis with ultraviolet radiation? A review of the literature. Arch Dermatol. 1991;127:1708-13.

11. Lebwohl M, Menter A, Koo J, Feldman SR. Combination therapy to treat moderate to severe psoriasis. J Am Acad Dermatol. 2004;50:416-30.

12. Carrascosa JM, López-Estebaranz JL, Carretero G, Daudén E, Ferrándiz C, Vidal D, et al. Narrowband UV-B, monochromatic excimer laser, and photodynamic therapy in psoriasis: A consensus statement of the Spanish Psoriasis Group. Actas Dermosifiliogr. 2011;102:175-86.

13. Penven K, Leroy D, Verneuil L, Faguer K, Dompmartin A. Evaluation of vaseline oil applied prior to UVB TLo1 phototherapy in the treatment of psoriasis. Photodermatol Photoimmunol Photomed. 2005;21:138-41.

14. Menter A, Korman NJ, Elmets CA, Feldman SR, Gelfand JM, Gordon $\mathrm{KB}$, et al. Guidelines of care for the management of psoriasis and psoriatic arthritis. Section 5. Guidelines of care for the treatment of psoriasis with phototherapy and photochemotherapy. J Am Acad Dermatol. 2010;62:114-35.

15. Bagel J. LCD plus NB-UVB reduces time to improvement of psoriasis vs. NB-UVB alone. J Drugs Dermatol. 2009;8:351-7.

16. Jain VK, Jangra S, Aggarwal K. Comparative efficacy of narrow-band ultraviolet B phototherapy alone and its combination with topical 8-methoxypsoralen in psoriasis. Indian J Dermatol Venereol Leprol. 2010;76:666-70.

17. Thaçi D, Salgo R. Malignancy concerns of topical calcineurin inhibitors for atopic dermatitis: Facts and controversies. Clin Dermatol. 2010;28:52-6.

18. Kokelj F, Plozzer C, Torsello P. Reduction of UV-A radiation induced by calcipotriol in the treatment of vulgar psoriasis with oral psoralen plus UV-A. Arch Dermatol. 1997;133:668-9.

19. Bailey EE, Ference EH, Alikhan A, Hession MT, Armstrong AW. Combination treatments for psoriasis: A systematic review and meta-analysis. Arch Dermatol. 2012;148:511-22.

20. Ashcroft DM, Li Wan Po A, Williams HC, Griffiths CE. Combination regimens of topical calcipotriene in chronic plaque psoriasis: Systematic review of efficacy and tolerability. Arch Dermatol. 2000;136:1536-43.

21. Brands S, Brakman M, Bos JD, de Rie MA. No additional effect of calcipotriol ointment on low-dose narrow-band UVB phototherapy in psoriasis. J Am Acad Dermatol. 1999;41:991-5.

22. Adachi Y, Uchida N, Matsuo T, Horio T. Clinical effect of vitamin D3 analogues is not inactivated by subsequent UV exposure. Photodermatol Photoimmunol Photomed. 2008;24:16-8.

23. Guerra A. Novedades en el tratamiento tópico de la psoriasis. Med Cutan iber Lat Am. 2004;32:79-89.

24. Behrens S, Grundmann-Kollmann M, Peter RU, Kerscher M. Combination treatment of psoriasis with photochemotherapy and tazarotene gel, a receptor-selective topical retinoid. $\mathrm{Br} \mathrm{J}$ Dermatol. 1999;141:177. 
25. Tzaneva S, Hönigsmann H, Tanew A, Seeber A. A comparison of psoralen plus ultraviolet A (PUVA) monotherapy, tacalcitol plus PUVA and tazarotene plus PUVA in patients with chronic plaque-type psoriasis. Br J Dermatol. 2002;147:748-53

26. Koo JY, Lowe NJ, Lew-Kaya DA, Vasilopoulos AI, Lue JC, Sefton J, et al. Tazarotene plus UVB phototherapy in the treatment of psoriasis. J Am Acad Dermatol. 2000;43:821-8.

27. Guenther LC. Optimizing treatment with topical tazarotene. Am J Clin Dermatol. 2003;4:197-202.

28. Paul C, Gallini A, Archier E, Castela E, Devaux S, Aractingi S, et al. Evidence-based recommendations on topical treatment and phototherapy of psoriasis: Systematic review and expert opinion of a panel of dermatologists. J Eur Acad Dermatol Venereol. 2012;26(Suppl.3):1-10.

29. Schiener R, Behrens-Williams SC, Pillekamp H, Kaskel P, Peter RU, Kerscher M. Calcipotriol vs. tazarotene as combination therapy with narrowband ultraviolet B (311 nm): Efficacy in patients with severe psoriasis. Br J Dermatol. 2000;143:1275-8.

30. Rogers C. Calcipotriol (Dovobet) ointment in combination with UVB therapy for psoriasis treatment. Dermatol Nurs Dermatol Nurses Assoc. 2006;18:258-61.

31. Arechalde A, Saurat JH. Management of psoriasis: The position of retinoid drugs. BioDrugs Clin Immunother Biopharm Gene Ther. 2000;13(5):327-33. Encontré: BioDrugs. 2000;13:327-33.

32. Spuls PI, Rozenblit M, Lebwohl M. Retrospective study of the efficacy of narrowband UVB and acitretin. J Dermatol Treat. 2003;14(Suppl.2):17-20.

33. Ghasri P, Yentzer BA, Dabade TS, Feldman SR. Acitretin for the treatment of psoriasis: An assessment of national trends. J Drugs Dermatol. 2011;10:873-7.

34. Dunn LK, Gaar LR, Yentzer BA, O’Neill JL, Feldman SR. Acitretin in dermatology: A review. J Drugs Dermatol. 2011;10:772-82.

35. Hodulik SG, Zeichner JA. Combination therapy with acitretin for psoriasis. J Dermatol Treat. 2006;17:108-11.

36. Mortazavi H, Khezri S, Hosseini H, Khezri F, Vasigh M. single blind randomized clinical study: The efficacy of isotretinoin plus narrow band ultraviolet B in the treatment of psoriasis vulgaris. Photodermatol Photoimmunol Photomed. 2011;27:159-61.

37. Ormerod AD, Campalani E, Goodfield MJD, BAD Clinical Standards Unit. British Association of Dermatologists guidelines on the efficacy and use of acitretin in dermatology. Br J Dermatol. 2010;162:952-63.

38. De Mozzi P, Johnston GA, Alexandroff AB. Psoriasis: An evidence-based update. Br J Dermatol. 2012;166:252-60.

39. Patel RV, Clark LN, Lebwohl M, Weinberg JM. Treatments for psoriasis and the risk of malignancy. J Am Acad Dermatol. 2009;60:1001-17.

40. Warren RB, Griffiths CEM. Systemic therapies for psoriasis: methotrexate, retinoids, and cyclosporine. Clin Dermatol. 2008;26:438-47.

41. Jensen P, Skov L, Zachariae C. Systemic combination treatment for psoriasis: A review. Acta Derm Venereol. 2010;90:341-9.

42. Shehzad T, Dar NR, Zakria M. Efficacy of concomitant use of PUVA and methotrexate in disease clearance time in plaque type psoriasis. J Pak Med Assoc. 2004;54:453-5.
43. Mahajan R, Kaur I, Kanwar AJ. Methotrexate/narrowband UVB phototherapy combination vs. narrowband UVB phototherapy in the treatment of chronic plaque-type psoriasis--a randomized single-blinded placebo-controlled study. J Eur Acad Dermatol Venereol. 2010;24:595-600.

44. Asawanonda P, Nateetongrungsak Y. Methotrexate plus na rrowband UVB phototherapy versus narrowband UVB phototherapy alone in the treatment of plaque-type psoriasis: A randomized, placebo-controlled study. J Am Acad Dermatol. 2006;54:1013-8.

45. Barrios M, Beltrán A, Catellanos H, Cortés C. Guías basadas en la evidencia para el manejo de la psoriasis en Colombia. Bogotá: Asociación Colombiana de Dermatología y Cirugía Dermatológica; 2012

46. Chalela JG, González C, Castro L. Guías de manejo de psoriasis consenso colombiano. Bogotá: Asociación Colombiana de Dermatología y Cirugía Dermatológica; 2008.

47. Echeverri MA, Aristizábal AM, Vargas F, Molina JF, Pinto LF Zuluaga Á. Nuevos avances de la terapia biológica en la psoriasis. Med Cutan Iber Lat Am. 2005;33:7-17.

48. Wolf P, Hofer A, Weger W, Posch-Fabian T, Gruber-Wackernagel A, Legat FJ. $311 \mathrm{~nm}$ ultraviolet B-accelerated response of psoriatic lesions in adalimumab-treated patients. Photodermatol Photoimmunol Photomed. 2011;27:186-9.

49. Bagel J. Adalimumab plus narrowband ultraviolet B light phototherapy for the treatment of moderate to severe psoriasis. J Drugs Dermatol. 2011;10:366-71.

50. Garber C, Creighton M, Sorensen E, Dumont N, Gottlieb AB. Systemic treatment of recalcitrant pediatric psoriasis: A case series and literature review. J Drugs Dermatol. 2015;14:881-6.

51. Famenini S, Wu JJ. Combination therapy with tumor necrosis factor inhibitors in psoriasis treatment. Cutis. 2013;92:140-7.

52. Scheinfeld N. Therapy resistant psoriasis treated with alefacept and subsecuent narrow band ultraviolet B phototherapy with total clearing of psoriasis. Dermatol Online J. 2005;11:7-11.

53. Koo J, Bagel J, Sweetser M, Ticho B. Alefacept in combination with ultraviolet B phototherapy for the treatment of chronic plaque psoriasis: Resulst from an open label, multicenter study. J Drugs Dermatol. 2006;5:623-8.

54. Bailey EE, Ference EH, Alikhan A, Hession MT, Armstrong AW. Combination treatments for psoriasis: A systematic review and meta-analysis. Arch Dermatol. 2012;148:511-22.

55. Menter A, Gottlieb A, Feldman SR, van Voorhees AS, Leonard CL, Gordon KB, et al. Guidelines of care for the management of psoriasis and psoriatic arthritis. Section 1. Overview of psoriasis and guidelines of care for the treatment of psoriasis with biologics. J Am Acad Dermatol. 2008;58:826-50.

56. Foley P, Quirk C, Sullivan J, Dolianitis C, Hack SP, Thirunavukkarasu $\mathrm{K}$, et al. Combining etanercept with traditional agents in the treatment of psoriasis: A review of the clinical evidence. J Eur Acad Dermatol Venereol. 2010;24:1135-43.

57. Chien AL, Elder JT, Ellis CN. Ustekinumab. A new option in psoriasis therapy. Drugs. 2009;69:1141-52.

58. Wolf P, Weger W, Legat FJ, Posch-Fabian T, Gruber-Wackernagel $\mathrm{A}$, Inzinger $\mathrm{M}$, et al. Treatment with 311-nm ultraviolet $B$ enhanced response of psoriatic lesions in ustekinumabtreated patients: A randomized intraindividual trial. $\mathrm{Br}$ Dermatol. 2012;166 :147-53. 